\title{
APOLLO: A new push in solar-system tests of gravity
}

\author{
T. W. Murphy, Jr. ${ }^{1}$, E. G. Adelberger ${ }^{2}$, J. B. R. Battat ${ }^{3}$, C. D. Hoyle ${ }^{4}$, \\ R. J. McMillan ${ }^{5}$, E. L. Michelsen ${ }^{1}$, C. W. Stubbs and H. E. Swanson ${ }^{2}$ \\ ${ }^{1}$ University of California, San Diego, \\ 9500 Gilman Drive, La Jolla, CA, 92093-0424, USA \\ email: tmurphy@physics.ucsd.edu \\ ${ }^{2}$ University of Washington, Seattle, WA 98195-1560, USA \\ ${ }^{3}$ Massachusetts Institute of Technology, Cambridge, MA 02139, USA \\ ${ }^{4}$ Humboldt State University, Arcata, CA 95521-8299, USA \\ ${ }^{5}$ Apache Point Observatory, Sunspot, NM 88349-0059, USA
}

\begin{abstract}
Lunar laser ranging (LLR) has long provided many of our best measurements on the fundamental nature of gravity, including the strong equivalence principle, time -rate-of-change of the gravitational constant, the inverse square law, geodetic precession, and gravitomagnetism. This paper serves as a brief overview of APOLLO: a recently operational LLR experiment capable of millimeter-level range precision.
\end{abstract}

Keywords. gravitation

\section{Introduction}

Lunar laser ranging (LLR) has long provided the best constraints on a number of tests of gravity, including the strong equivalence principle, time variation of the gravitational constant, geodetic precession, the inverse square law, and gravitomagnetism (Williams et al. 1996; Murphy et al. 2007; Soffel et al. 2008).

APOLLO (the Apache Point Observatory Lunar Laser-ranging Operation) represents a new capability in the technique of lunar laser ranging (LLR), achieving millimeter-level range precision. Beginning in 2006, this order-of-magnitude improvement over previous capabilities will translate into similar gains in tests of general relativity, and provide a more comprehensive understanding of solar system dynamics as well as probe the interior structure of the moon.

What is required to reach a $1 \mathrm{~mm}$ goal? The random uncertainty associated with the lunar range measurement is dominated by the temporal spread induced by the finite-sized retroreflector trays tilted by angles up to 10-deg by lunar librations, amounting to 15$50 \mathrm{~mm}$ of one-way range error for an individual photon. Improvements in range precision must, therefore, come about by increasing the number of photons that constitute the range measurement. Improvements in laser pulse width, timing precision, etc. do not result in greater range precision. To reduce the $15-50 \mathrm{~mm}$ uncertainty introduced by the reflector, one must collect 225-2500 photons, which far exceeds typical performance of previous LLR stations. APOLLO, by virtue of its deployment on a large $3.5 \mathrm{~m}$ telescope at a site with good atmospheric seeing, is able to measure thousands of photon round trip times in a matter of minutes, exceeding previous records by a factor of $\sim 70$. This boost in sensitivity is what allows APOLLO to push LLR into the millimeter regime.

A detailed description of the APOLLO science motivation, instrument design, and initial performance may be found in Murphy et al. (2008). Demonstration of one-millimeter 
Table 1. APOLLO record performance for the different reflectors.

\begin{tabular}{l|c|c|c|c}
\hline Reflector & photons per run & photons per minute & $\begin{array}{c}\text { photons per shot } \\
(5 \text { minute average })\end{array}$ & $\begin{array}{c}\text { photons per shot } \\
(15 \text { second average })\end{array}$ \\
\hline Apollo 11 & $4497(26 \times)^{1}$ & $1079(65 \times)$ & 0.90 & 1.4 \\
\hline Apollo 14 & $7606(36 \times)$ & $1825(69 \times)$ & 1.52 & 2.0 \\
\hline Apollo 15 & $15740(26 \times)$ & $3775(67 \times)$ & 3.15 & 4.5 \\
\hline Lunokhod 2 & $750(11 \times)$ & $180(31 \times)$ & 0.15 & 0.24 \\
\hline
\end{tabular}

Notes:

${ }^{1}$ Ratios in parentheses denote the factor by which APOLLO exceeds previous records.

ranging performance is provided in Battat et al. (2009). In this paper, we present a few results not published elsewhere as a demonstration of APOLLO's promise to transform lunar ranging.

\section{APOLLO Performance}

APOLLO seeks to bring about an order-of-magnitude improvement in tests of gravity by way of a substantial enhancement in the rate of return photon detection. Not only are the random uncertainties tamed by the gain in $\sqrt{N}$, but systematic errors may be investigated in much greater detail when the signal is more robust. For example, strong runs may be split into shorter segments to look for scatter beyond that expected from random error, as was done in Battat et al. (2009). Model systematics may be checked by cycling among the various reflectors multiple times within a session-exposing lunar orientation offsets. Once confidence has been established in the short-term behavior of the system (within a one-hour session), any systematic drift in residuals may be used to inform earth orientation offsets.

Table 1 demonstrates APOLLO's photon collection capabilities. Though each run may vary in duration, the records represented in the first column all come from runs consisting of 5000 shots, lasting 250 seconds. The second column normalizes to photons collected in a 1 minute period. Here we see that APOLLO outperforms the previous records (in parentheses: all previous records set by the French LLR station at Grasse) by a factor of $\sim 65$ for all three Apollo reflectors. Lunokhod 2 is less responsive than it was in the past.

The higher return rate achieved by APOLLO means that we may obtain ranges at full moon for the first time since the McDonald $2.7 \mathrm{~m}$ telescope performed LLR measurements prior to 1985. This is especially important for the Equivalence Principle measurement, to which full moon measurements are most sensitive.

As an example of how the high return rate can impact model systematics, Fig. 1 shows the measured range offset from a prediction based on the DE421 ephemeris from the Jet propulsion Laboratory (JPL). The nine data points cover four reflectors over a 45 minute period. The four reflector residuals can be fit individually by a common slope, which is due mostly to inadequate treatment of earth orientation on the APOLLO prediction software, and may thus be ignored. The offset between the four reflectors indicates imperfect knowledge of the lunar orientation, as predicted in the DE421 ephemeris. An obvious coherence exists in the APOLLO data, so that the individual measurements are selfconsistent within the error bars, allowing for arbitrary lunar orientation. Subtracting each reflector's individual fit-line puts all the residuals on the same footing, as seen in Fig. 2. The distribution of residuals is artificially small with relation to the error bars because the lunar orientation was "fixed" to minimize the residuals rather than appealing to a physical model of the moon subject to fitting requirements of data points on other nights. 


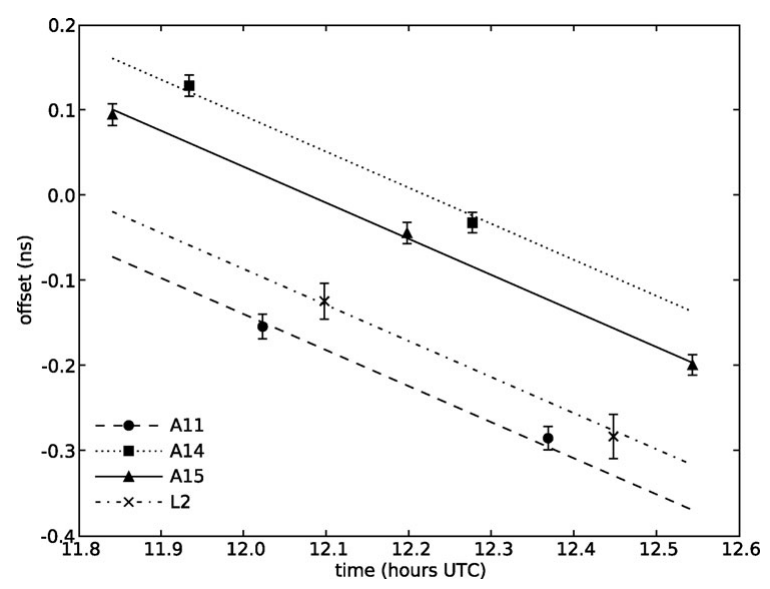

Figure 1. APOLLO residuals from the DE421 ephemeris on the night of 2008 September 24 . $0.1 \mathrm{~ns}$ in the round-trip time is equivalent to $15 \mathrm{~mm}$ of one-way range. A common slope offset applies to all reflectors, indicating a likely earth orientation offset. The separation of the four reflectors is due to imperfect prediction of lunar orientation.

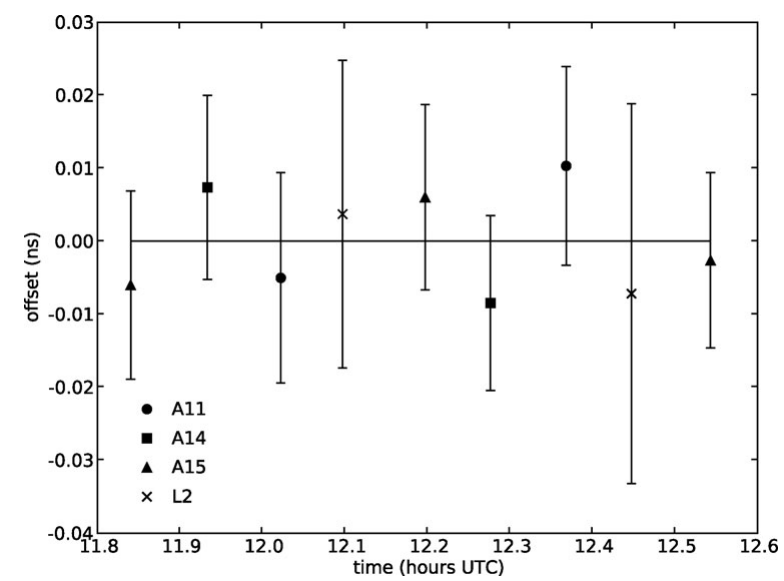

Figure 2. Residuals from Fig. 1 after subtracting the individual fit-lines for each reflector. The remaining offsets are artificially small with relation to the error bars because five degrees of freedom have been removed from 9 points (a common slope and four individually-adjusted offsets). Each vertical tick of $0.01 \mathrm{~ns}$ corresponds to $1.5 \mathrm{~mm}$ in one-way range.

Though Figures 1 and 2 represent a particular night, no deviation from this behavior has yet been seen in APOLLO data. Reflector-specific offsets are common, but the data points coherently follow a common trend-line among the reflectors. At the very least, then, APOLLO data points can be expected to have substantial power in establishing lunar orientation. As this is crucial for converting ranges to individual reflectors into effective ranges to the center of the moon, this capability should deliver also a direct improvement on the gravitational physics measured by LLR.

The overall residuals produced from APOLLO data points using the best models available do not yet indicate fidelity between model and observation. There could be many contributing reasons, and it is difficult to separate systematic measurement errors from model shortcomings. Thus far, the tests we can easily perform have not shown any obvious problems with data quality, but this is only possible over short timescales. Meanwhile, we know the models lack small-scale influences like ocean and atmospheric loading of the 
earth crust, earth center-of-mass motion, and the latest models on atmospheric refraction. Also, APOLLO data are not yet used at full weight to influence earth orientation solutions. It is, therefore, imperative that we advance the state-of-the-art in LLR modeling, so that we may use APOLLO's high data quality and advance the limits of our knowledge about gravitational physics.

\section{References}

Battat, J. B. R., Murphy, T. W., Adelberger, E. G., Gillespie, B., Hoyle, C. D., McMillan, R. J., Michelsen, E. M., Nordtvedt, K., Orin, A. E., Stubbs, C. W., \& Swanson, H. E. 2009, PASP 121, 29

Murphy, T. W., Nordtvedt, K., \& Turyshev, S. G. 2007, Phys. Rev. Lett., 98, 071102

Murphy, T. W., Adelberger, E. G., Battat, J. B. R., Carey, L. N., Hoyle, C. D., LeBlanc, P., Michelsen, E. M., Nordtvedt, K., Orin, A. E., Strasburg, J. D., Stubbs, C. W., Swanson, H. E., \& Williams, E. 2008, PASP 120, 20

Soffel, M., Klioner, S., Müller, J., \& Biskupek, L. 2008, Phys. Rev. D, 78, 024033

Williams, J. G., Newhall, X. X., \& Dickey, J. O. 1996, Phys. Rev. D, 53, 6730 